\title{
An Investigation of Engineering Design Cognition and Achievement in Primary School
}

\author{
Greg J. Strimel \\ Purdue University \\ Scott R. Bartholomew \\ Brigham Young University - Provo, scottbartholomew@byu.edu \\ Eunhye Kim \\ Purdue University \\ Liwei Zhang \\ Purdue University \\ Follow this and additional works at: https://scholarsarchive.byu.edu/facpub \\ Part of the Educational Assessment, Evaluation, and Research Commons, Elementary Education \\ Commons, Engineering Education Commons, Instructional Media Design Commons, Other Education \\ Commons, and the Other Engineering Commons
}

\section{Original Publication Citation}

Strimel, G. J., Bartholomew, S. R., Kim, E., \& Zhang, L. (2018). An Investigation of Engineering Design Cognition and Achievement in Primary School. Journal of STEM Education Research, $1(1), 173-201$.

\section{BYU ScholarsArchive Citation}

Strimel, Greg J.; Bartholomew, Scott R.; Kim, Eunhye; and Zhang, Liwei, "An Investigation of Engineering Design Cognition and Achievement in Primary School" (2018). Faculty Publications. 5552.

https://scholarsarchive.byu.edu/facpub/5552

This Peer-Reviewed Article is brought to you for free and open access by BYU ScholarsArchive. It has been accepted for inclusion in Faculty Publications by an authorized administrator of BYU ScholarsArchive. For more information, please contact ellen_amatangelo@byu.edu. 
See discussions, stats, and author profiles for this publication at: https://www.researchgate.net/publication/328597294

\section{An Investigation of Engineering Design Cognition and Achievement in Primary School}

Article in Journal for STEM Education Research · December 2018

DOI: 10.1007/541979-018-0008-0

CITATIONS

11

4 authors, including:

Greg J. Strimel

Purdue University

111 PUBLICATIONS 434 CITATIONS

SEE PROFILE

Eunhye Kim

Purdue University

25 PUBLICATIONS 75 CITATIONS

SEE PROFILE

Some of the authors of this publication are also working on these related projects:

Adaptive Comparative Judgment to Inform and Assess Design View project

Advancing Excellence in P-12 Engineering Education (AEEE) Project View project
413

Scott Bartholomew

Brigham Young University - Provo Main Campus

64 PUBLICATIONS 274 CITATIONS

SEE PROFILE 


\title{
An Investigation of Engineering Design Cognition and Achievement in Primary School
}

\author{
Greg J. Strimel ${ }^{1}$ (D) Scott R. Bartholomew ${ }^{1} \cdot$ Eunhye Kim $^{1} \cdot$ Liwei Zhang $^{1}$
}

Published online: 29 October 2018

(C) Springer Nature Switzerland AG 2018

\begin{abstract}
This study examined the design cognition and achievement results of both kindergarten and fourth grade students engaged in engineering design-based instructional activities. Relationships between design cognition and student grade level, as well as quality of student work, were investigated. 30 concurrent think-aloud protocols were collected from individual primary students as they worked in groups to design and make a solution to a design task. The concurrent think-aloud protocols were examined and coded to determine the duration of time the participants devoted to a pre-established set of mental processes for technological problem solving. Significant differences between kindergarten and fourth grade participants were found in the amount of time various cognitive processes were employed. Fourth grade students dedicated significantly more time to the mental processes of Creating, Defining Problems, Measuring, and Testing than kindergarten students. In addition, when examining the think-aloud protocols along with the evaluations of the participant's design work, it was found that more time devoted to the cognitive process of Managing could be a significant predictor of lower design achievement. These findings can highlight potential areas for improving educational practice based on the cognitive abilities of students at different grade levels and the quality of their design work. As engineering design-based activities become more prevalent for the teaching of STEM-related content and practices, the results of this research, and the employed methodology, may demonstrate a promising practice for better understanding and assessing such education efforts.
\end{abstract}

Greg J. Strimel

gstrimel@purdue.edu

Scott R. Bartholomew

sbartho@purdue.edu

Eunhye Kim

kim1906@purdue.edu

Liwei Zhang

zhan1128@purdue.edu

1 Purdue University, West Lafayette, IN, USA 
Keywords Primary school - Engineering education · Design cognition · Design assessment - Concurrent think-aloud protocols

\section{Introduction}

Engineering design activities, which require students to work in groups to devise, test, optimize, and communicate a solution to an open-ended problem, have been a hallmark of engineering education for decades (Cunningham 2009; Dutson et al. 1997; Engineering Accreditation Commission 2016; National Academy of Engineering and National Research Council 2009). More recently, the interest in engineering education for P-12 students has been on the rise (Carr et al. 2012; Strimel et al. 2016), and the presence of engineering design activities in primary schools has increased (Hegedus 2014). At the primary level, engineering design-based learning is believed to help young students expand their often-limited perceptions of engineering. According to Cunningham and Hester (2007), young students often perceive engineering as just using, fixing, and/or improving things which is void of the practices related to informed design (Grubbs and Strimel 2015). Moreover, engineering design activities are often used to help students foster twenty-first century skills, such as teamwork and collaboration, as they work together in open-ended design environments (Hammack et al. 2015). These activities are also believed to support students in learning the practices necessary to frame problems, plan and develop solutions, and share ideas with others (McCullar 2015). Studies have indicated that engineering activities for children can indirectly influence their learning and achievement in science and mathematics (National Academy of Engineering and National Research Council 2009). In the long term, exposure to engineering education has been found to be influential to students in developing career aspirations for engineering and other STEM-related occupations (Capobianco et al. 2012; Hegedus 2014; National Academy of Engineering and National Research Council 2009). As a result, multiple attempts have been made to integrate engineering education into primary school curricula. For example, curriculum programs such as Engineering is Elementary from the Boston Museum of Science, Launch from Project Lead the Way, and the Technology, Engineering, Environment, Mathematics, and Science (TEEMS) by Engineering byDesign ${ }^{\mathrm{TM}}$ can be found in schools across the country. In addition, engineering design has become a core component of the standards for both technology education (ITEA/ITEEA, 2000/2002/2007) and science education (NGSS Lead States 2013) in the United States.

In this context, it becomes important to assist educators in using developmentally appropriate curriculum and pedagogical strategies to ensure students are able to build capabilities to employ engineering design practices to produce viable solutions to authentic problems. However, engineering design can be challenging to understand, teach, and evaluate as many efforts to infuse engineering are void of empirical foundations around how students think as they engage in engineering design (Dym et al. 2005). Therefore, examining engineering design cognition at various levels of education has become a topic of interest to STEM education practitioners and researchers in hopes to provide recommendations for effective teaching, the suitable scaffolding of engineering design experiences, and ultimately, enhancing student design abilities (Lammi and Becker 2013). However, minimal agreement exists on how primary students design and limited examinations have explored more effective 
ways to bridge design cognition research with teaching and learning strategies (Grubbs 2013). Moreover, there has been limited research investigating design cognition at the primary level (Kelley et al. 2015). These concerns may be further exacerbated, as most research does not examine design cognition with respect to the evaluation of the outcomes of both designing and making. Much of design cognition research is limited to only identifying the cognitive processes involved with developing an initial solution concept without the creation of a prototype. While previous design cognition studies have been beneficial for identifying procedures in which participants employ to develop design ideas, the lack of research relating their cognitive activity to the tangible outcomes of their design process may (a) lead researchers to overlook the mental strategies involved in making and optimizing prototypes and (b) limit opportunities to uncover potential design heuristics for improved design achievement.

As Atman and Bursic (1998) explain, examining both design process and product can enable the uncovering of potential relationships between a student's thinking and the outcomes of their process. They further describe that understanding this potential relationship can help to recognize successful or unsuccessful practices in engineering design. As described by Wilson et al. (2013), this type of research may provide useful heuristics for teachers who seek to incorporate engineering practices within their classrooms. Therefore, related research efforts could support the development of instructional interventions aimed toward improving the teaching of engineering design and developing effective problem solvers. As a result, the authors conducted a study to investigate the cognitive activities of both kindergarten and fourth grade students as they designed and prototyped a solution to an engineering design task. The research was conducted with the intent of comparing student's design processes with the evaluation of their design work to highlight potential indicators for improving design practice. In addition, the research design facilitated the comparison of these results with students' grade levels. In doing so, the findings presented in this article can potentially help identify ways in which to better scaffold the teaching and learning of engineering design at the primary level.

\section{Design Cognition Research Review}

In tandem with the increased emphasis on engineering design, at all levels of education, attempts to establish effective ways of teaching the practices of engineering design have similarly increased (Dym et al. 2005). As part of these efforts, engineering design cognition studies have been conducted across multiple disciplines and professions (Grubbs and Strimel 2016; Lammi and Gero 2011). Design cognition studies commonly employ a concurrent think-aloud protocol procedure which is used to capture a participant's actions, along with their own verbal interpretation of their thought processes as they perform those actions, while solving a predetermined design task (Kelley et al. 2015). The resulting verbal interpretations are then analyzed using a verbal protocol analysis technique. This technique involves segmenting the collected design protocol into individual cognitive tasks and then applying a previously derived coding scheme over each segment of either a video recording or transcription of a design session (Purcell et al. 1996). The coded data can then be used to interpret the processes and procedures a participant employs to develop a solution to the design task. However, much of this type of research may provide an incomplete picture of design cognition, as limited studies examine participants beyond the development of a design concept, 
through the making of a prototype. Therefore, an incomplete understanding of the cognitive processes involved in both designing and making may exist which can also limit the means for comparing a participant's design process with the assessment of their process. Further, many of these studies collect student data from group-settings; which, although beneficial, captures group discussions and limits collecting an individual's thought process(es) while designing. While both settings (i.e., individual and group) are worthy of study, this effort focuses on individual student cognitive processes while engaged in a design task. Therefore, the participants were individually equipped with point-of-view cameras and trained to think aloud while working within their groups to complete the design tasks. As such, only the individual thought processes of the participants wearing the cameras were collected and then coded solely from their own perspective.

In addition to the previously highlighted research gaps, design cognition studies have traditionally focused on how engineers, architects, or post-secondary engineering students solve an engineering design problem (Cross 2001; Grubbs 2013; Lammi and Becker 2013). However, as the emphasis on P-12 engineering education continues to increase (Grubbs and Strimel 2016), the body of P-12 engineering design cognition studies has been growing with the purpose of understanding and improving pupils' engineering design thinking capabilities. Through a review of literature, Grubbs et al. (2018) identified 15 published design cognition studies involving unique data collected from P-12 students between 1995 and 2016. Of these studies, 14 investigated the design cognition of secondary students (grades 6-12) while only one study (Kelley et al. 2015) examined how primary students (grades K-5) distributed their cognitive processes when solving engineering design problems.

Kelley et al. (2015) studied the design thinking of students in grades five and six while they engaged in engineering design tasks. As the researchers examined the collected thinkaloud protocols, they determined that students in these grades were able to transfer the learning of the systematic approaches to engineering design — such as problem identification, analysis, and refinement of design solutions - to subsequent design contexts. However, their analysis indicated that fifth and sixth grade students might not fully understand, appreciate, or value the iterative nature of design, as several students did not conduct multiple iterations. These researchers also found that participants tended to emphasize the brainstorming of design solutions. Therefore, they posited that teaching students how to frame design problems and manage brainstorming sessions are critical to students' success as designers. Also, Kelley et al. (2015) suggested that more research using the concurrent think-aloud protocol procedure should be conducted to further investigate how primary students learn and how teachers use design experiences to enhance learning.

\section{Statement of Problem}

While the teaching of engineering design continues to increase in primary schools, uncertainty exists as to how students, at various grade levels, navigate a multifaceted engineering design problem. Specifically, limited research is available that examines how students at the primary education level distribute their cognitive efforts while completing an engineering design task. Additionally, little research exists that examines cognition during both the designing and making of a solution for primary students. With greater insight to design cognition, educators may be 
better equipped to manage the difficulties in planning and assessing student abilities for producing viable solutions to engineering design problems and researchers can examine the relationships between student design thinking across grade levels. Hence, the purpose of this study was to identify the cognitive processes employed by kindergarten and fourth grade students to solve engineering design problems in an effort to expand the understanding of how these pupils cognitively navigate an engineering design process from design conception through the production of a prototype. In addition, this research was intentionally designed and conducted in such a manner to compare a student's thinking process with the evaluation of their design work to enable the investigation of potential significant cognitive predictors of success in designing and making.

Addressing this research gap can be of specific importance as engineering design activities have now become widespread within primary school coursework - specifically following the introduction of engineering design within the Next Generation Science Standards (NGSS Lead States 2013) and along with the continued implementation of national engineering curriculum programs within primary schools. While theories about cognitive development, such as Piaget's Cognitive Development Theory, can suggest that children might be unable to operate in an ill-structured design space, others (Cohen 2002; Crossland 2015; Sutherland 1992; Weiten 1992) have contended that these theories miscomprehend the development of children as their learning capabilities and biological maturation can vary widely. According to these incongruities, the authors believe that exploring how kindergarteners and fourth grade students cognitively navigate open-ended design tasks may shed light on primary student design thinking and assist in identifying potentially-useful pedagogical approaches for improving student achievement through the better scaffolding of design activities.

\section{Research Objectives and Questions}

This study was driven by the overarching objectives of (1) identifying ways in which primary students cognitively navigate an engineering design task from design conception through prototype production and (2) determining ways in which to inform P-12 engineering education by examining design cognition in respect of student grade level and design achievement. To achieve these objectives the following research questions were generated:

Research Objective 1: identify ways in which primary students cognitively navigate an engineering design task from design conception through prototype production.

$\mathbf{R Q}_{1 \mathbf{a}}$ : What cognitive processes do kindergarten students employ to design and make solutions to engineering design problems?

$\mathbf{R Q}_{\mathbf{1 b}}$ : What cognitive processes do fourth grade students employ to design and make solutions to engineering design problems? 
Research Objective 2: determine ways in which to inform P-12 engineering education by examining design cognition in respect of student grade level and design achievement.

$\mathbf{R Q}_{\mathbf{2} \mathbf{a}}$ : What differences, if any, exist between the design cognition of kindergarten and fourth grade students?

$\mathbf{R Q}_{\mathbf{2} \mathbf{b}}$ : What are the potential cognitive indicators, if any, of design achievement for kindergarten and fourth grade students?

\section{Methods}

\section{Participants and Design Tasks}

Data from 30 concurrent think-aloud protocols (18 kindergarten participants, ages 5-7, and 12 fourth grade participants, ages 8-10) were collected across four classrooms (2 kindergarten, 2 fourth grade) while students engaged in one of six design tasks - that were similarly structured but contextually different. The data collection took place in one small suburban school district located in the Midwestern United States during the spring of 2017. At the time, the district was composed of a mainly middle-class population and served approximately 10,000 students with only a small percentage (22\%) of those receiving free/reduced meals. Following the receipt of Institutional Review Board approval for research with minors, four teachers (two fourth grade teachers, two kindergarten teachers) from one primary school in this district were recruited for participation based on their STEM integration efforts involving engineering design activities. Each teacher was recommended by the school instructional excellence coach and had similar years of experience, licensure qualifications, and experience implementing engineering design activities in their classroom. All teachers were Caucasian, had been teaching for more than $5 \mathrm{yrs}$, and had only just begun integrating engineering design tasks into their classrooms. Prior to the study, each teacher was trained on the various design activities and a member of the research team was present during each class to ensure fidelity of implementation throughout the study.

Each teacher in this study led their class through three open-ended engineering design activities (see Table 1), which involved students working in groups to employ an "elementary level" design process (steps including; Ask, Explore, Model, Evaluate, and Explain) to resolve the posed problems. The students, in each grade level, were presented with a design problem from a book currently being read and discussed in class [e.g., Pink and Say by Patricia Polacco (1994) for fourth grade students and My Bug Box by Pat Blanchard and Suhr (1999) for kindergarten students] and asked how they might solve the problem highlighted in the literature [e.g., How can we create a device to help carry a wounded soldier (Pink and Say) or How can we create a box to keep bugs safe from the toad (My Bug Box)?]. Each task required the students to design and make a physical prototype/model while also completing a design worksheet to document their process. Students worked in teacher-assigned groups of 2-3 to identify the criteria and constraints for the presented problem from the in-class reading, explore 
Table 1 Design challenges

\begin{tabular}{|c|c|c|}
\hline Grade & Design Task & In Class Reading \\
\hline Kindergarten & $\begin{array}{l}\text { How can we keep the bugs safe from the toad? } \\
\text { - Design and build a bug box that does not allow } \\
\text { frogs in but allows bug in/out }\end{array}$ & $\begin{array}{l}\text { My Bug Box by Pat Blanchard } \\
\text { and Suhr (1999) }\end{array}$ \\
\hline Kindergarten & $\begin{array}{l}\text { How can we help the dragonfly's legs that were } \\
\text { unable to catch prey? } \\
\text { - Design and build a device to help the dragonfly } \\
\text { catch prey }\end{array}$ & Dragonflies by Margaret Hall (2006) \\
\hline Kindergarten & $\begin{array}{l}\text { How can we help toad come out during the day? } \\
\text { - Design and build a device to help the toad come } \\
\text { out into the sunlight without getting too hot }\end{array}$ & Toads by Alyse Sweeney (2010) \\
\hline 4th Grade & $\begin{array}{l}\text { How can we help Pink to carry Say? } \\
\text { - Design and build a device to help carry a } \\
\text { wounded soldier }\end{array}$ & $\begin{array}{l}\text { Pink and Say by Patricia } \\
\text { Polacco (1994) }\end{array}$ \\
\hline 4th Grade & $\begin{array}{l}\text { How can we conceal a secret message? } \\
\text { - Design and build a device to conceal and carry a } \\
\text { secret message for the army }\end{array}$ & $\begin{array}{l}\text { Great Women of the Civil War } \\
\text { by Molly Kolpin (2014) }\end{array}$ \\
\hline 4th Grade & $\begin{array}{l}\text { How can we make Civil War soldiers stay healthy } \\
\text { and strong? } \\
\text { - Design and build a device to help protect soldiers }\end{array}$ & $\begin{array}{l}\text { The Terrible, Awful Civil War } \\
\text { by Kay Mechisedech (2011) }\end{array}$ \\
\hline
\end{tabular}

important questions, brainstorm ideas, develop possible solutions, and evaluate the effectiveness of their solution. As each group attempted to solve the problem, they completed the design worksheet (see Fig. 1), which was developed collaboratively by the teachers and researchers involved in this project. To complete the challenge, the students were provided with several low-fidelity prototyping materials such as craft sticks, straws, paper, masking tape, etc. to produce their solutions. Each design session centered on one problem and lasted $31 \mathrm{~min}$ on average. While working on the problem, students were allowed to move freely about the classroom, solicit help from peers or the teacher, and obtain additional supplies, if needed, with permission from the teacher. A sample of the student prototypes and completed design worksheets are shown in Fig. 2.

\section{Concurrent Think-Aloud Protocol Collection and Analysis}

This study employed a multiple exploratory case study approach using concurrent think-aloud protocol analysis to identify the cognitive processes used by both kindergarten and fourth grade participants as they worked to develop solutions to engineering design problems. Atman and Bursic (1998), when studying undergraduate students, posited that using concurrent think-aloud protocol analysis was a valuable process for understanding the cognitive processes students use when developing a design solution. Although different in age, and development, the authors postulate that younger students will be similarly capable of using concurrent think-aloud protocol. This position is based upon the rationale for the use of think-aloud methods with triads of primary students that was developed by Kelley et al. (2015). They state that concurrent think-aloud approaches can serve as an easier method for engaging young participants in eliciting their thought processes 


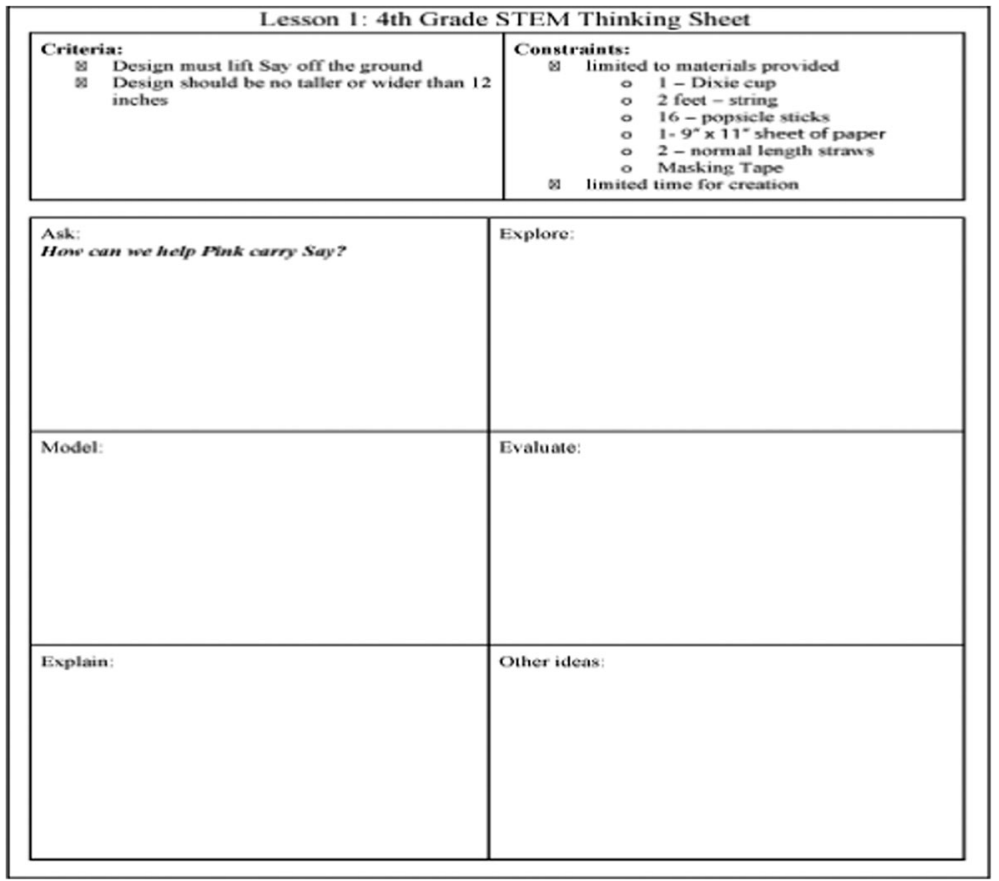

Fig. 1 Example design worksheet for one of the assigned design tasks

as it enables them to use their own language within their own dialog, communicate their design thoughts in a genuine way rather than through a more structured elicitation technique, removes the risk of the researcher imposing upon their natural thought processes, and allows them to express their design thinking while engaged in the act of designing.
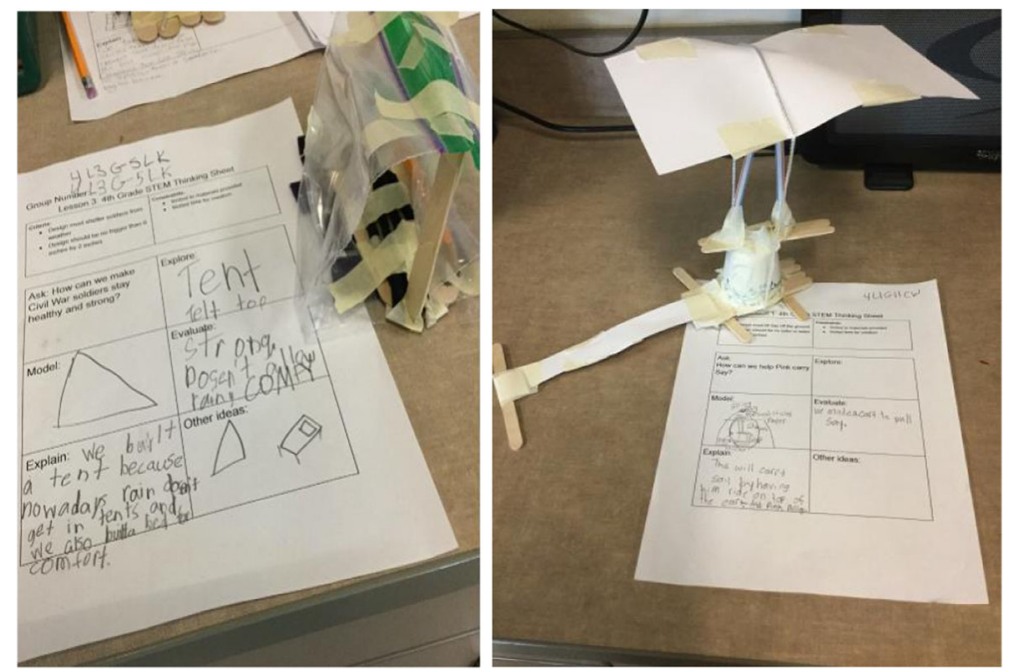

Fig. 2 Student design worksheet and prototype examples (Left: 4th grade, Design and build a device to help protect soldiers; Right: 4th grade, Design and build a device to help carry a wounded soldier) 
Accordingly, the participants in this study were asked to verbalize their thoughts while engaged in an engineering design task and, to facilitate data collection, they were equipped with point-of-view cameras that enabled the recording of verbal protocols as well as their non-verbal cues-known as observational protocol. The combination of think-aloud and observational protocols in addition to the participants' design artifacts were used in data triangulation during the data analyses (Cross 2004). While students in these classes were required to work in groups for each design task, the concurrent think-aloud protocols were collected from one participant per group. These participants were selected by the teachers based on their comfort with wearing the point-of-view cameras - which is recognized as a limitation to this study. Once the participants were selected, following the approved Institutional Review Board protocol, and equipped with the cameras, they were tasked to think aloud while completing the design task.

Following data collection, the recorded think-aloud protocols were segmented into individual utterances and coded using the 17 mental processes for technological problem solving defined and validated by Halfin (1973). This coding scheme was selected as it was developed through a content analysis of practicing designers, innovators, technologists, and engineers and validated through a Delphi study by Halfin (1973) and revalidated by Wicklein and Rojewski (1999). The operational definitions of these 17 mental processes and sample utterances are provided in Table 2. However, based on a review of literature, the mental process of Modeling was determined by the researchers to be too similar to the other codes of Model/Prototype Constructing and Designing. The inability to differentiate between these codes was stated in the original work by Halfin (1973). As a result, Modeling was not used and the actions that could be considered Modeling were coded as either Designing or Model/Prototype Constructing. Also, it is important to note that the mental processes identified by Halfin (1973) may not all be represented in the thought processes of young learners as they may beyond their level of development. While this can be a limitation to this study, the results of this research can highlight the mental processes that may not be developmentally appropriate to expect with the different ages of the learners.

Additionally, to further clarify coding decisions, enhance transparency, and support replication, this study organized a hierarchy (see Fig. 3) of the16 cognitive processes based on a study by Strimel (2014) which highlighted patterns of cognitive processes within a conceptual engineering design model. According to his study, the processes of Communicating and Managing are often employed throughout an entire engineering design task to control and converse the actions and outcomes of the problem-solving episode. Next, the processes of Analyzing, Designing, Creating, Model/Prototype Constructing, and Testing are predominately employed during a particular phase of the design process. Lastly, the mental processes of Experimenting, Observing, Computing, Measuring, Interpreting Data, Questioning/Hypothesizing, Predicting, Defining Problems, and Visualizing are often employed parsimoniously throughout an engineering design task. Therefore, this study assigned Communicating and Managing as level 1 codes; Analyzing, Designing, Creating, Model/Prototype Constructing, and Testing as level 2 codes; and Experimenting, Observing, Computing, Measuring, Interpreting Data, Questioning/Hypothesizing, Predicting, Defining Problems, and Visualizing as level 3 codes. These codes were layered to assist with coding specifically when 


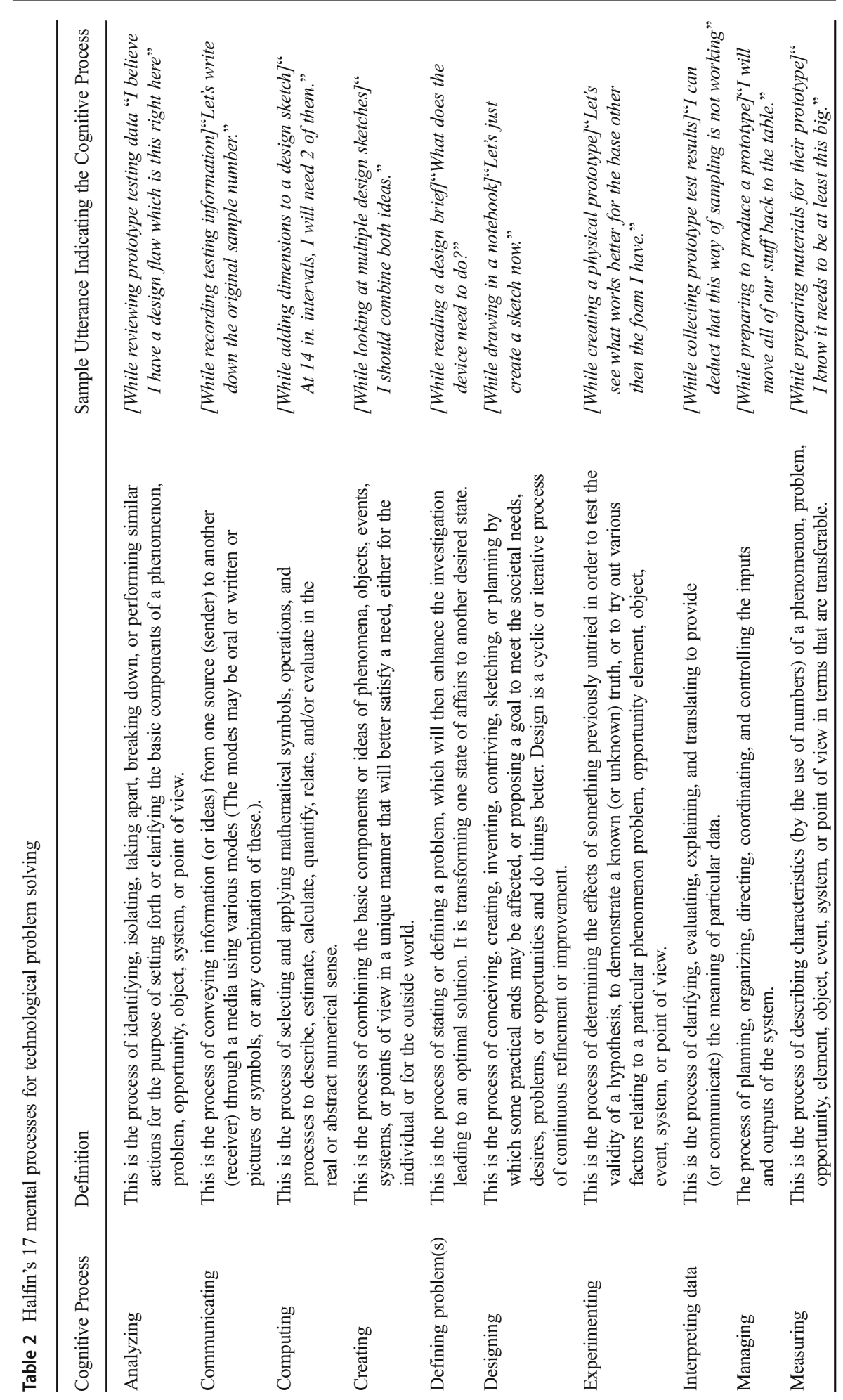




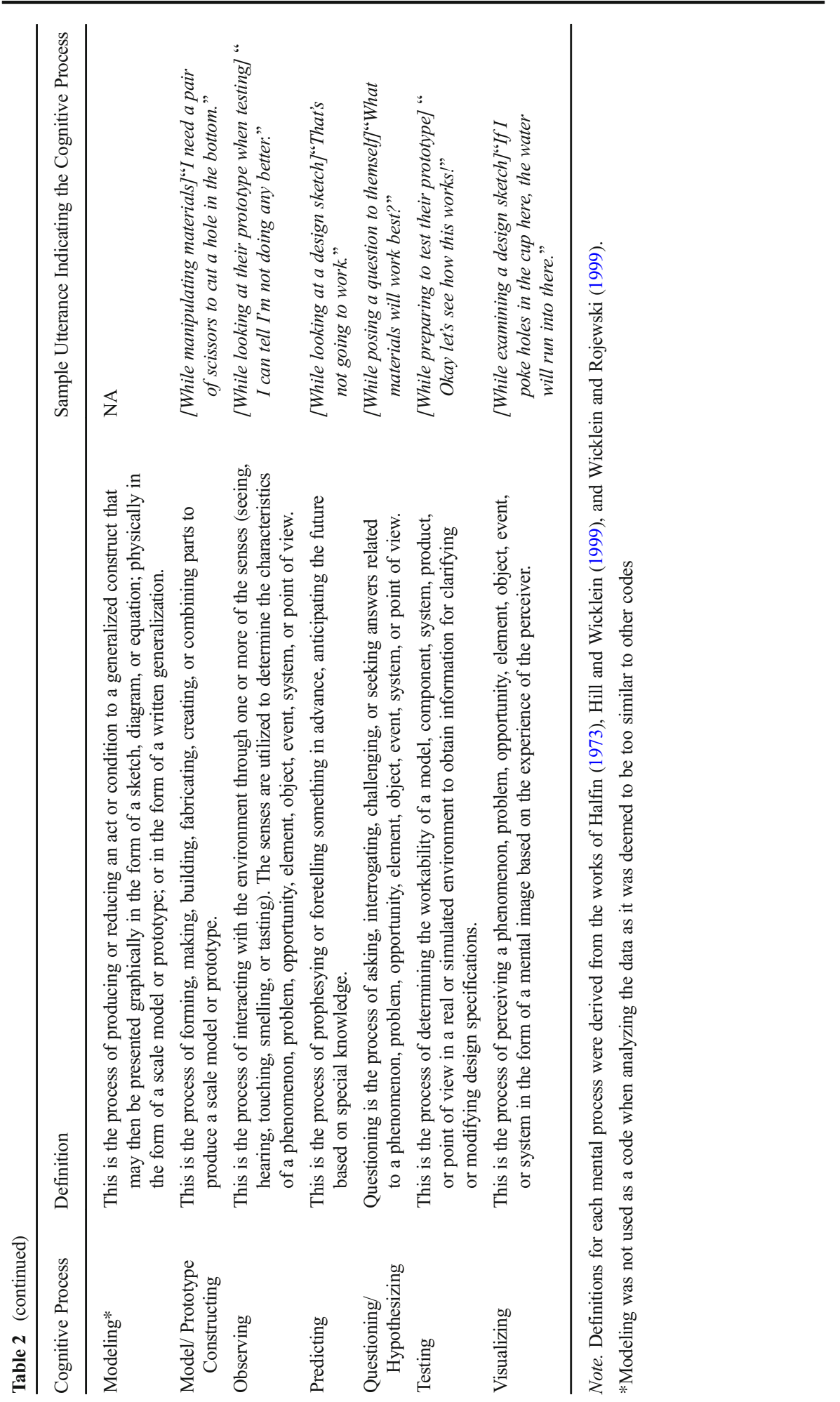




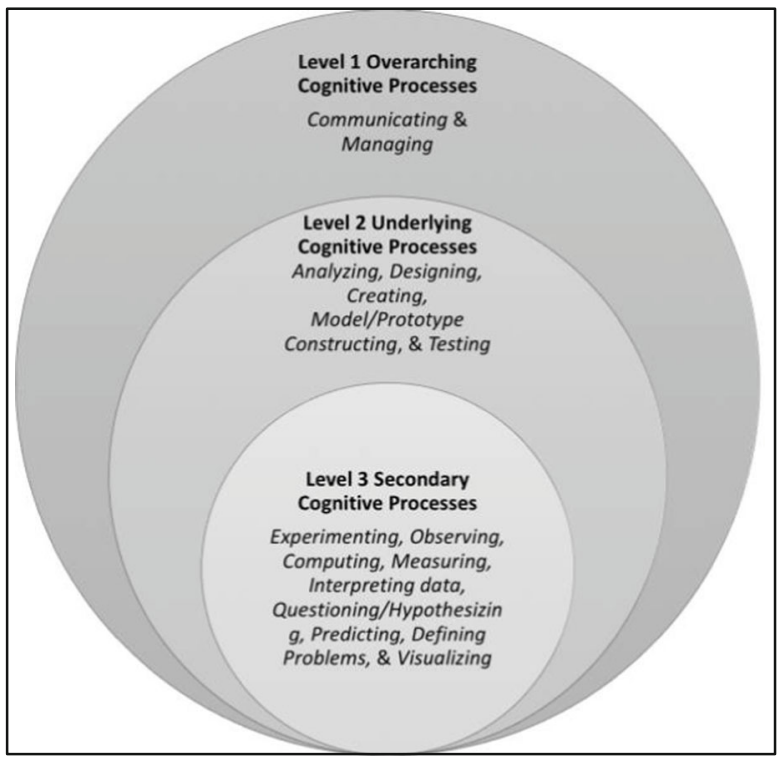

Fig. 3 Hierarchy of Halfin's mental processes for the purpose of guiding the coding of design protocols

multiple cognitive processes were thought to be occurring simultaneously. These levels do not indicate complexity as each process can potentially have its own levels of complexity. However, based on the analysis of previous design protocols and design cognition research, the order of these codes indicates what cognitive process could be considered at the forefront of the participants' thoughts. Therefore, in the hierarchy of the 16 codes, priority for a coding decision, where multiple processes could be considered as happening simultaneously, was given to the higher level codes as they were seen as the most relevant to the actions of the participant.

To enable the coding process, the researchers used NVivo software, which permits a researcher to segment and code the recorded protocols simultaneously while observing the video recordings (Mentzer et al. 2015). Also, to ensure the reliability of this procedure, two independent coders were involved in the coding process (Mentzer 2014). After each coder coded each segment of every protocol, agreement rates and Cohen's Kappa values were calculated between the two coders' results (Cohen 2007). Across all codes for the three design projects for each grade level, the average of agreement rates was $97.67 \%$, and the average of Cohen's Kappa values was 0.72, which indicates substantial agreement of the analysis (McHugh 2012).

\section{Design Achievement Analysis}

The design worksheets and prototypes were evaluated by the teachers using the Adaptive Comparative Judgment (ACJ) assessment technique - a method that has recently gained attention as a reliable and valid method of evaluating open-ended design problems (Bartholomew 2017; Bartholomew et al. 2017; Kimbell 2012; Seery et al. 2016; Bartholomew et al. 2018). Through the ACJ software CompareAssess, teachers assessed students design portfolios at the conclusion of the assignment (the portfolios consisted of the design worksheet and an image of their prototype). This ACJ 
process involved teachers viewing two student portfolios and, rather than assigning a grade through micro-scoring using a rubric, they were asked to bear in mind the assignment criteria and holistically chose which portfolio was better. The specific assignment criteria, which was provided to teachers and students from the onset of the assignment and which was used by teachers in making their paired comparative judgments, was (1) demonstration of collaboration, (2) design process, and (3) the final prototype creation.

Recognizing that this approach to assessment was novel to these teachers, a training session was held prior to the research to acquaint them with the method. Further, poststudy interviews with teachers revealed that they were comfortable with the approach and felt confident in the results produced. ACJ, as an approach to assessment has repeatedly demonstrated an improved ability to achieve very high reliability levels over traditionally used approaches, such as rubrics, as the process centers on comparative decisions rather than a series of subjective decisions (e.g., based on multiple criteria from a rubric). In this study, as the teachers progressed through a series of paired comparisons, choosing the better design portfolio each time, an embedded algorithm using rash-model statistics produced a rank order of all included student work. The rank order provides a potentially better means for analyzing achievement over scores collected through a rubric. When using rubrics, teachers can assign multiple students similar scores while their design actions/outcomes may be different. However, a rank order provides a defined sequence of achievement from the best to the worst based on the collective consensus of those judging the work. Therefore, the resulting rank order from the ACJ process was used to represent participants' design achievement and applied in the subsequent data analysis related to the research questions.

\section{Findings}

\section{Research Objective 1: Identify how Primary Students Cognitively Navigate an Engineering Design Task}

\section{$\mathrm{RQ}_{1 \mathrm{a}}$ : What Cognitive Processes do Kindergarten Students Employ to Design and Make} Solutions to Engineering Design Problems? This question was answered by coding video recordings of participants thinking aloud during engineering design tasks. On average, kindergarten participants completed the tasks within $30 \mathrm{~min}$ and $45 \mathrm{~s}$. Throughout the engineering design activities, the top three most employed mental processes were Model/Prototype Constructing, Managing, and Observing. Model/Prototype Constructing consumed $34.4 \%$ of the participant's time on average, which mostly consisted of physically manipulating materials for making prototypes. Next, Managing consumed $20.7 \%$ of the participant's time on average and consisted mostly of finding/contemplating materials and tools, planning next steps, coordinating priorities, and directing a teammate to do something. Lastly, Observing consumed $13.3 \%$ of the participant's time on average and consisted mostly of examining/handling materials and watching the actions of a teammate or a situation to understand the phenomenon.

After coding the design protocols, it was determined that the least used mental processes for the kindergarten participants were Testing $(0.1 \%)$, Creating $(0.3 \%)$, Measuring (0.4\%), Defining Problems (0.6\%), Questioning (1.2\%), and Predicting (1.2\%); each of these mental processes consumed less than $2 \%$ of the participant' time 
on average. Of note, the mental processes of Computing and Experimenting were not observed with the kindergarten participants. The average time dedicated to each cognitive process throughout the design task and standard deviation can be found in Table 3. Figure 4 provides a graphical representation of the division of time dedicated to each cognitive process, on average, throughout the design tasks.

\section{$\mathrm{RQ}_{1 \mathrm{~b}}$ : What Cognitive Processes do Fourth Grade Students Employ to Design and Make}

Solutions to Engineering Design Problems? This question was answered by coding video recordings of participants thinking aloud during engineering design tasks. On average, fourth grade participants completed the tasks within $27 \mathrm{~min}$ and $32 \mathrm{~s}$. Throughout the design activities, the top three most used mental processes were Model/Prototype Constructing, Managing, and Analyzing. Model/Prototype Constructing consumed $26.3 \%$ of the fourth-grade participant's design time on average, followed by Managing, which consumed $18.0 \%$ of their time, and Analyzing, which consumed $17.9 \%$ of the participant's time on average. The Analyzing time consisted mostly of gathering and examining information related to the design problem, listening to peers and teachers to understand design decisions, and clarifying possible explanations of a phenomenon.

After coding the design protocols, it was determined that the least used mental processes for the fourth grade participants were Creating (1.0\%), Visualizing (1.3\%),

Table 3 Mean cognitive process distribution for the engineering design activities

\begin{tabular}{|c|c|c|c|c|c|c|}
\hline & \multicolumn{2}{|c|}{$\begin{array}{l}\text { Kindergarten Participants } \\
\left(N_{K}=18\right)\end{array}$} & \multicolumn{2}{|c|}{$\begin{array}{l}\text { 4th Grade Participants } \\
\left(N_{4 t h}=12\right)\end{array}$} & \multicolumn{2}{|c|}{$\begin{array}{l}\text { Total Participants } \\
(N=30)\end{array}$} \\
\hline & $\begin{array}{l}\text { Mean } \\
(\text { Sec. })\end{array}$ & $\begin{array}{l}\text { Standard } \\
\text { Deviation } \\
\text { (Sec.) }\end{array}$ & $\begin{array}{l}\text { Mean } \\
(\mathrm{Sec} .)\end{array}$ & $\begin{array}{l}\text { Standard } \\
\text { Deviation (Sec.) }\end{array}$ & $\begin{array}{l}\text { Mean } \\
(\text { Sec. })\end{array}$ & $\begin{array}{l}\text { Standard } \\
\text { Deviation } \\
\text { (Sec.) }\end{array}$ \\
\hline Analyzing & 183.73 & 104.45 & 259.93 & 200.03 & 214.21 & 151.70 \\
\hline Communicating & 116.68 & 64.39 & 109.69 & 42.71 & 113.89 & 55.99 \\
\hline Computing & 0.00 & 0.00 & 0.00 & 0.00 & 0.00 & 0.00 \\
\hline Creating & 4.36 & 6.45 & 14.36 & 11.57 & 8.36 & 10.00 \\
\hline Designing & 66.36 & 53.52 & 87.33 & 51.62 & 74.74 & 52.91 \\
\hline Defining Problems & 9.72 & 11.35 & 40.98 & 35.25 & 22.22 & 28.10 \\
\hline Experimenting & 0.00 & 0.00 & 0.00 & 0.00 & 0.00 & 0.00 \\
\hline Interpreting Data & 0.38 & 1.63 & 0.00 & 0.00 & 0.23 & 1.26 \\
\hline Managing & 315.28 & 145.40 & 261.01 & 95.17 & 293.57 & 128.68 \\
\hline Measuring & 6.35 & 10.63 & 19.57 & 16.26 & 11.64 & 14.49 \\
\hline Model/Prototype & 523.22 & 218.62 & 382.33 & 223.22 & 466.87 & 227.70 \\
\hline Observing & 202.14 & 146.57 & 155.58 & 98.66 & 183.52 & 129.70 \\
\hline Predicting & 18.91 & 30.25 & 21.50 & 22.89 & 19.95 & 27.15 \\
\hline Questioning & 17.79 & 17.99 & 36.92 & 37.04 & 25.44 & 28.30 \\
\hline Testing & 1.16 & 4.93 & 45.56 & 64.89 & 18.92 & 45.83 \\
\hline Visualizing & 53.85 & 54.95 & 19.29 & 15.87 & 40.03 & 46.50 \\
\hline Total Time & 1844.23 & 532.27 & 1651.08 & 402.51 & 1766.97 & 486.61 \\
\hline
\end{tabular}




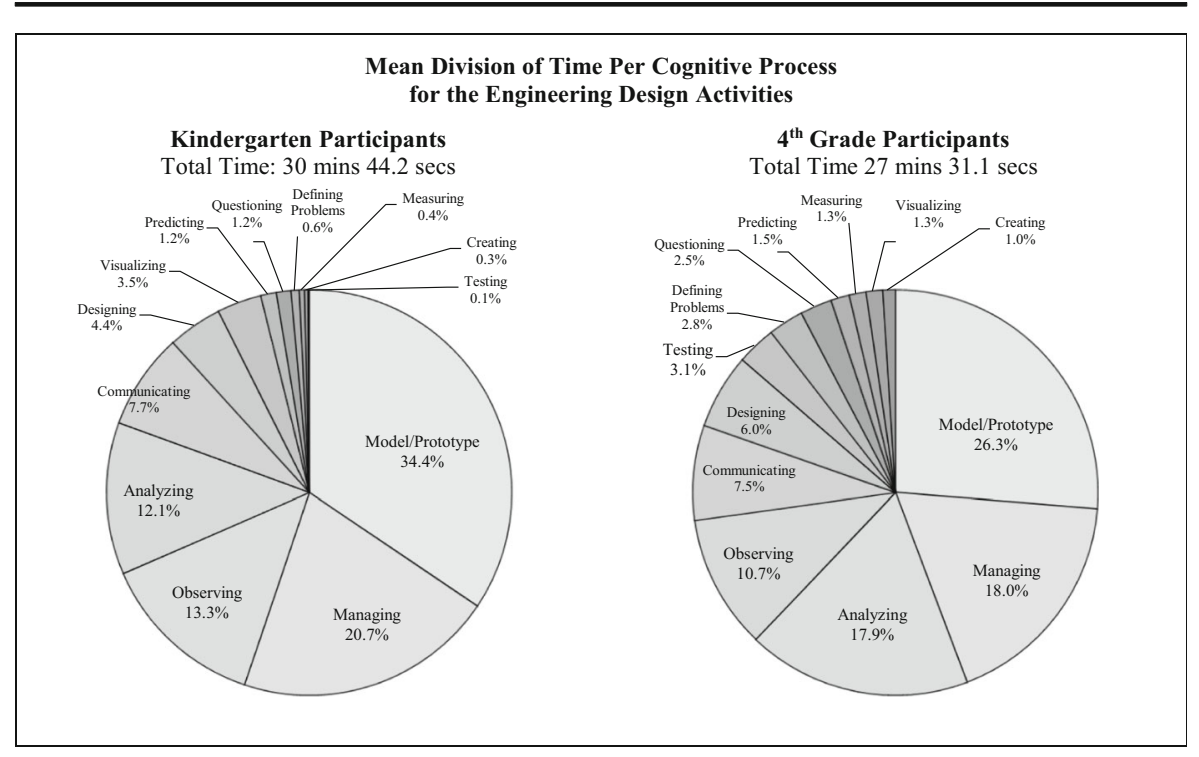

Fig. 4 Mean division of time dedicated to each cognitive process, on average, throughout the design tasks

Measuring (1.3\%), and Predicting (1.5\%). Each of these mental processes consumed less than $2 \%$ of the participant' time on average. Of note, similar to the kindergarten students, the mental processes of Computing and Experimenting were not observed with the fourth grade participants. The mean time dedicated to each cognitive process throughout the design task and standard deviation can also be found in Table 3. Figure 4 includes a graphical representation of the division of time dedicated to each cognitive process, on average, throughout the design tasks.

\section{Research Objective 2: Examine Design Cognition in Respect of Student Grade Level and Design Achievement}

$\mathrm{RQ}_{2 \mathrm{a}}$ : What Differences, if any, Exist between the Design Cognition of Kindergarten and Fourth Grade Students? To address this question, the researchers performed a comparison between kindergarten and fourth grade participants' design cognition. Before choosing a method for the comparison, the researchers tested normality and the homogeneity of variance assumptions of the data. The test results demonstrated violation of these assumptions. Therefore, recognizing that the Mann-Whitney test is less sensitive to these violations, the researchers applied the non-parametric test for comparing the two groups. The Mann-Whitney test indicated that there was no significant difference in the total time used for designing between the kindergarten and fourth grade participants. However, the test results did indicate that fourth grade participants devoted significantly more time to Creating (Kindergarten $M=4.36 \mathrm{~s}$, Fourth grade $M=14.36 \mathrm{~s}, U=50.00, p=.011$ ), Defining Problems (Kindergarten = $9.72 \mathrm{~s}$, Fourth grade $=40.98 \mathrm{~s}, U=51.00, p=.015$ ), Measuring (Kindergarten $M=$ $6.35 \mathrm{~s}$, Fourth grade $M=19.57 \mathrm{~s}, U=52.50, p=.012$ ), and Testing (Kindergarten $M=$ $1.16 \mathrm{~s}$, Fourth grade $M=45.56 \mathrm{~s}, U=49.50, p=.001)$ than the kindergarten 
participants. The greatest difference in cognitive processing time between the two groups was found in the mental process of Testing (Kindergarten $M=1.16 \mathrm{~s}$; Fourth grade $M=45.56 \mathrm{~s}$ ). Although most kindergarten participants ended their design session once a prototype was made, the fourth grade participants revealed the design practices of Testing, which was demonstrated by examining the workability and/or durability of their prototypes and then attempting to improve the prototypes. The complete MannWhitney analysis results are presented in Table 4.

$\mathrm{RQ}_{2 \mathrm{~b}}$ : What Are the Potential Cognitive Indicators, if any, of Design Achievement for Kindergarten and Fourth Grade Students? To address this question, the researchers examined the correlations between the achievement of the 30 participants and the cognitive processes they revealed during the design session. The achievement was determined by the teachers' evaluations of the participants' project deliverables (prototypes and design worksheets) using the ACJ assessment technique, which resulted in a rank order of student projects. The analysis between the rank orders and cognitive processing times of the primary students revealed that project rankings were significantly correlated with the amount of time dedicated to the cognitive process of Managing (Spearman's rho $=.442, p=.014$ ). This finding indicates that a project's rank tended to increase (meaning the project's quality decreased) as participants

Table 4 Mann-Whitney analysis between kindergarten and fourth grade participants

\begin{tabular}{|c|c|c|c|c|}
\hline & \multicolumn{2}{|c|}{$\begin{array}{l}\text { Mean Time Dedicated to Each Cognitive } \\
\text { Process (sec.) }\end{array}$} & \multirow[t]{2}{*}{ Mann-Whitney U } & \multirow[t]{2}{*}{$\begin{array}{l}\text { Asymp. Sig. } \\
\text { (2-tailed) }\end{array}$} \\
\hline & $\begin{array}{l}\text { Kindergarten } \\
(N=18)\end{array}$ & $\begin{array}{l}4^{\text {th }} \text { Grade } \\
(N=12)\end{array}$ & & \\
\hline Analyzing & 183.73 & 259.93 & 88.00 & 0.397 \\
\hline Communicating & 116.68 & 109.69 & 104.00 & 0.866 \\
\hline Computing & 0.00 & 0.00 & N/A & N/A \\
\hline Creating & 4.36 & 14.36 & 50.00 & $0.011^{*}$ \\
\hline Designing & 66.36 & 87.33 & 79.00 & 0.220 \\
\hline Defining Problems & 9.72 & 40.98 & 51.00 & $0.015^{*}$ \\
\hline Experimenting & 0.00 & 0.00 & N/A & N/A \\
\hline Interpreting Data & 0.38 & 0.00 & 102.00 & 0.414 \\
\hline Managing & 315.28 & 261.01 & 83.00 & 0.290 \\
\hline Measuring & 6.35 & 19.57 & $\mathbf{5 2 . 5 0}$ & $0.012 *$ \\
\hline Model/Prototype & 523.22 & 382.33 & 71.00 & 0.117 \\
\hline Observing & 202.14 & 155.58 & 94.00 & 0.553 \\
\hline Predicting & 18.91 & 21.50 & 91.00 & 0.466 \\
\hline Questioning & 17.79 & 36.92 & 64.00 & 0.063 \\
\hline Testing & 1.16 & 45.56 & 49.50 & $0.001 * *$ \\
\hline Visualizing & 53.85 & 19.29 & 66.00 & 0.075 \\
\hline Total Time & 1844.23 & 1651.08 & 93.00 & 0.525 \\
\hline
\end{tabular}

**Significant at the 0.01 level (2-tailed)

*Significant at the 0.05 level (2-tailed) 
dedicated more time to the process of Managing. During the design session, participants had to allocate their given time to multiple cognitive strategies, so if they spent a certain amount of time on a cognitive process, then they may lose the amount of time for other, maybe more critical cognitive processes. Thus, the results may suggest that students need to use their time Managing more efficiently and distribute their cognitive efforts to other metal strategies to achieve better project results. Based on the observations of participants and the limited time they devoted to the process of Defining Problems, the authors found that a lack of problem framing and scoping may have resulted in the increased time needed for Managing their design efforts. As such, efforts to assist students in effectively Defining Problems may be beneficial.

Besides Managing, there were no statistically significant correlations between participants' assessment results and any other cognitive processes employed during the design session. However, Creating, Defining Problems, Interpreting Data, Measuring, Observing, Predicting, Testing, and Visualizing were all correlated with better ranks, although these relationships were not statistically significant. It should be noted that a negative correlation suggests a positive relationship between cognitive process and achievement as a lower rank signifies a better achievement level. Table 5 presents the correlation analysis results between cognitive processes and participant achievement.

Table 5 Correlation between participants' cognitive processes and design achievement

\begin{tabular}{|c|c|c|}
\hline & \multicolumn{2}{|l|}{ ACJ Rank } \\
\hline & Spearman's rho & $\begin{array}{l}\text { Sig. (2-tailed) } \\
\mathrm{p}\end{array}$ \\
\hline Analyzing & .083 & .661 \\
\hline Communicating & .045 & .813 \\
\hline Computing & N/A & N/A \\
\hline Creating & -.108 & .572 \\
\hline Designing & .109 & .566 \\
\hline Defining Problems & -.161 & .397 \\
\hline Experimenting & N/A & N/A \\
\hline Interpreting Data & -.163 & .391 \\
\hline Managing & $.442^{*}$ & .014 \\
\hline Measuring & -.239 & .203 \\
\hline Model/Prototype & .127 & .504 \\
\hline Observing & -.168 & .374 \\
\hline Predicting & -.144 & .449 \\
\hline Questioning & .074 & .697 \\
\hline Testing & -.016 & .934 \\
\hline Visualizing & -.277 & .138 \\
\hline Total Time & .316 & .088 \\
\hline
\end{tabular}

The codes of Computing and Experimenting were not used by the participants

*Significant at the 0.05 level (2-tailed) 
Furthermore, to identify potential significant cognitive predictors of design success in terms of project rank, ordinal regression analyses of the design cognition data were calculated between the cognitive process times and the final project ranks. Prior to pursuing the ordinal regression analyses, regression diagnostics were conducted to test statistical assumptions of linearity, homoscedasticity, normality of residuals, mean independence, and non-linear relationships. These assumptions were met with the exception of an issue with multicollinearity related to several of the predictors (cognitive processes) being highly correlated with one another. To account for this issue, the number of collinear predictors was reduced by combining highly correlated cognitive processes into aggregate processes. Based on the results of these statistical diagnostics and the work by Strimel et al. (2018) the cognitive processes of Computing and Interpreting Data were combined to form Quantitative Reasoning; Experimenting, Testing, Questioning/Hypothesizing, and Observing were combined to form Scientific Inquiring; and Designing and Creating were combined to form Designing/Ideating (see Fig. 5). Following the creation of the new cognitive process codes, the data were again examined with the ordinal regression procedure to investigate potentially significant cognitive predictors for design achievement (rank). Based on the correlations between a total of 11 variables, two-way interaction terms significantly correlated with one another were included in the analysis, but there was no significant interaction effect. The results indicated that Managing was a significant predictor of project rank. An increase in time spent on Managing was associated with a poorer design ranking, with an odds ratio of .007 (95\% CI, .002 to .013), Wald $\chi 2(1)=6.239, p=.012$. Thus, in this sample a participant's rank would worsen by .007 of a rank for each second they devoted to the process of Managing. This result can be aligned with the result of

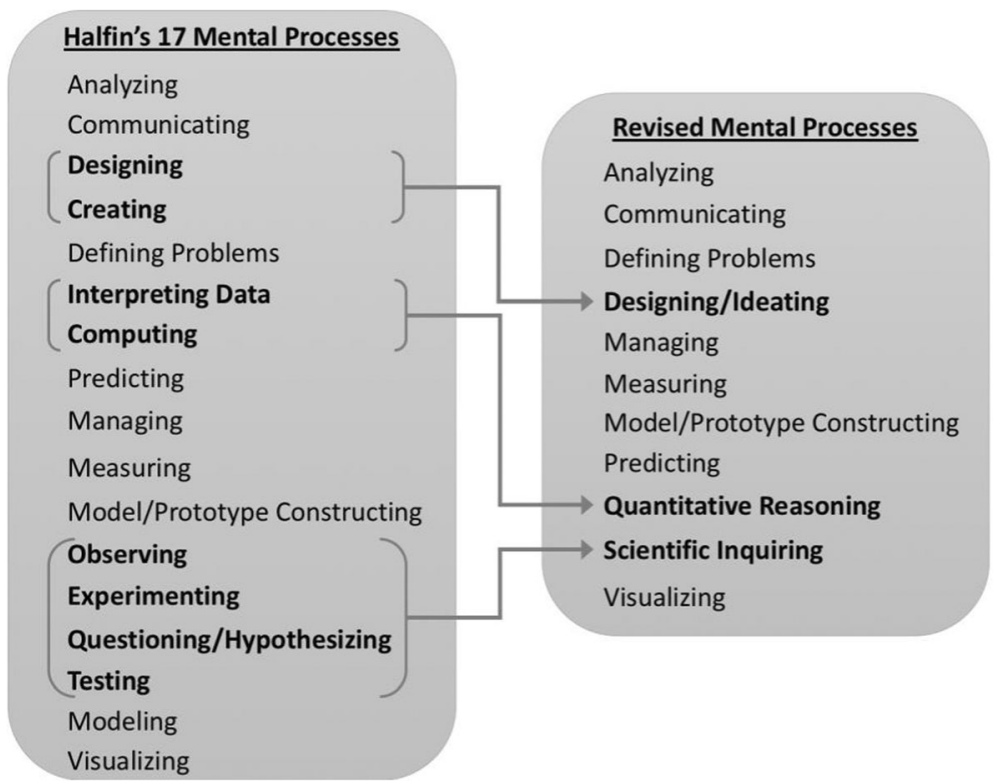

Fig. 5 Proposed revised list of the 17 mental processes for technological problem solving based on the combination of highly correlated processes. Note: Modeling was removed from the list based on Halfin's (1973) original work which highlighted that the process was too similar to other mental processes (Model/ Prototype Constructing and Designing) 
correlation analysis, which suggests that it could be important for students to develop abilities to effectively spend their time Managing by better framing design problems. These changes may provide more time for students to devote to other cognitive tasks that could potentially help them achieve better achievement results.

\section{Discussions and Recommendations}

It is important to note that this study was exploratory in nature and limited to a sample of 30 participants. Therefore, the results of this study are not generalizable to all primary school engineering programs. Although limitations exist, the findings can highlight elements of design cognition that warrant further investigation and provide discussion points/recommendations for pedagogical practice within the STEM education community. Accordingly, the following sections will discuss this study's findings and present recommendations for future research and educational practice.

\section{Design Cognition Research Recommendations}

Typically the rationale for conducting design cognition research is to better understand how different groups of people solve design problems. However, researchers should be purposeful in their research design to help bridge the gap between design cognition research and educational practice in order to better inform teaching and learning. Therefore, this study attempted to discover the relationships, if any, between students' grade level, cognition, and success in engineering design through concurrent thinkaloud protocol, correlational, and ordinal regression analyses. Specific attention was focused on identifying potential cognitive predictors of achievement in design to provide a foundation for recommendations toward improving student design abilities. The analysis results showed that Managing was the only cognitive process correlated with the ranking of student design success. However, this relationship implied that the more time students devoted to Managing, the poorer their design portfolio was ranked. The authors believe that during the design session, participants had to allocate their given time to multiple cognitive strategies; and consequently, the more time they spent on Managing, the less time they were able to engage in other, potentially more critical, cognitive processes. These other cognitive processes may have positively impacted their design achievement. For example, while not significant, the mental processes of Creating, Defining Problems, Interpreting Data, Measuring, Observing, Predicting, Testing, and Visualizing were all related to a better design ranking. However, these were some of the least used cognitive processes by the participants. Thus, the results may imply that interventions could be developed, such as improved problem framing practices or interim goal setting, in an attempt to help primary students use their Managing time more efficiently by better defining design problems and goals. As seen in this example, this type of research can help identify potential pain points in the design practices of students. Therefore, the authors recommend that the research methods presented in this study be enacted with larger sample sizes of students at various ages and experience levels within a variety of settings to further investigate the development of design practices and design-based educational interventions. 
Also, to help facilitate and provide consistency in the design protocol coding process, it is recommended for future researchers using the Halfin (1973) coding scheme to consider the hierarchical organization of the cognitive processes proposed by the authors in Fig. 3. This organization assisted the research team in making more consistent coding decisions throughout the data analysis process when more than one mental process seemed to be occurring at the same time. The organization enabled the selection of the code that was perceived to be the most relevant to the actions of the participant. Further, as several cognitive processes were highly correlated with one another in this sample and also in the study conducted by Strimel et al. (2018), there is support for potentially refining the Halfin (1973) coding scheme by combining highly correlated cognitive processes into aggregate processes (see Fig. 5). While a revised set of codes may not be suitable for all settings, future research focused on identifying cognitive predictors of success through an ordinal regression analysis, should consider combining the cognitive processes of Computing and Interpreting data to form Quantitative Reasoning; Experimenting, Testing, Questioning/Hypothesizing, and Observing to form Scientific Inquiring; and Designing and Creating to form Designing/Ideating.

Lastly, as stated by Kelley and Sung (2017), it is recommended that concurrent think-aloud protocol analysis be considered as a form of design assessment. In their study, they found that students were able to achieve success on traditional pre/post multiple-choice assessments developed to measure mathematical and scientific knowledge but the same students demonstrated flawed conceptual understandings of the topics when completing a design task. Therefore, Kelley and Sung (2017) believe that the concurrent think-aloud protocol analysis method may be an effective process for assessing a student's ability to transfer learning.

\section{Discussions for Informing Educational Practice}

Problem Framing and Scoping In this study, the analysis of 30 design protocols revealed a tendency for primary students to allocate the largest portion of the cognitive activity toward manipulating materials to produce a physical prototype rather than problem framing and scoping. While fourth grade students in this study dedicated significantly more cognitive effort to Defining Problems than kindergarten students, the two groups, overall, devoted limited time to analyzing and defining the given problems. In particular, the observations of kindergarten students suggested a shortcoming in ability to apply design criteria and constraints to their design. These findings can be of importance to educational practice as Strimel et al. (2018) found that higher design rubric scores on a design project were significantly correlated with the total time participants dedicated toward the design phase of their problem-solving process, which includes the practice of problem scoping. Additionally, Kelley et al. (2015) speculated that student teams who dedicated more time to defining problems could complete their problem-solving task more efficiently. Other studies have also indicated that the lack of effort in identifying design criteria and constraints can lead students to impulsively determine feasibilities of their solutions or to delay the investigation of alternative solutions later in their design process (Mentzer 2014; Mentzer et al. 2015; Wilson et al. 2013).

Strimel (2014) suggested that the lack of students' efforts to define problems may be a result of students' familiarity with solution-driven design approaches rather than problem-driven ones. Kruger and Cross (2006) explain that problem-driven design 
involves the designer focusing on understanding the given problem by carefully examining the design task to either (1) frame a well-defined problem to limit potential distractions from solution alternatives or (2) abstractly define a problem to provide opportunities for alternative solutions. In either case, they believe the problem-driven approach strongly focuses or concentrates the process of generating solutions. In contrast to this approach, Kruger and Cross (2006) describe that solution-driven design involves the designer keeping the problem ill-defined and focusing most of their efforts on generating a large number of potential solutions. When Kruger and Cross (2006) studied designers to compare these different design strategies, they found that those using a solution-driven strategy tended to have lower overall solution quality scores but higher creativity scores. However, they found that those using a problem-driven design strategy tended to produce the best results in terms of the balance of both overall solution quality and creativity. Accordingly, it may be necessary for primary students to have more opportunities to learn problem-driven approaches and practice transferring them to a variety of engineering design contexts.

Further emphasizing a need to examine the practices involved with problem framing, the primary students in this study were observed focusing attention on ancillary and irrelevant components of the design scenario. For example, kindergarten students tended to spend more time on making items, such as model insects for the project that required the creation of a device to help an injured dragonfly catch prey, instead of the actual prototype of the device to solve the problem. This was also observed with one of the fourth grade participants that spent most of their design time making model soldiers instead of a prototype shelter for the soldiers, which was the actual focus of the design problem. When associating these observations with the low percentage of cognitive processing time dedicated to Defining Problems (Kindergarten, 0.6\%; Fourth Grade, $2.8 \%$ ) when compared to Managing (Kindergarten, 20.7\%; Fourth Grade, 18.0\%) and Model/Prototype Constructing (Kindergarten, 34.4\%; Fourth Grade, 26.3\%), it appears that problem framing and scoping is a challenge for primary students and may not be developmentally appropriate for kindergarten students. As the findings suggest, more time associated with the cognitive process of Managing was a significant predictor of a poorer ranking and this process was often seen linked with the inability to understand or frame the problem. These findings also align with other reports (Berland 2013; Berland and Busch 2012; Goldstone and Sakamoto 2003; Kaminski et al. 2009; National Academy of Engineering and National Research Council 2014; Sloutsky et al. 2005) that describe how the rich perceptual information associated with, and irrelevant features of, design experiences can potentially distract students from identifying and learning the difficult to understand theoretical discipline-specific concepts. Therefore, when implementing engineering design tasks with young children, one should take cautionbased on their purpose for the design-based learning initiatives - as engineering design tasks are not proven to be the best method for developing transferrable knowledge in all situations and learning could be limited based on a student's problem framing abilities.

Furthermore, the findings related to problem framing and scoping also align with the results of a study by Welch and Lim (2000), which examined a total of 28 middle school students' design cognition. Their study determined that the majority of middle school students' cognitive efforts were used for modeling a possible solution idea and building a physical prototype. Additionally, the findings align with the studies conducted by Grubbs (2016) and Wilson et al. (2013) which indicate that secondary 
students tend to focus more on making physical solutions rather than clearly defining the design problems and analytically developing design concepts prior to prototyping. Moreover, Strimel et al. (2018) reported observing first-year engineering students with no prior engineering education experiences struggling to determine the necessary tasks to begin the design process when required to solve an ill-structured problem. Therefore, as the practices of (a) clearly defining goals associated with problem-solving efforts, (b) determining key information and procedures required to solve a problem, and (c) recognizing the constraints that limit potential solutions are necessary for achieving true engineering design capabilities (Cross 2001), these findings may provide support for establishing a coherent and better scaffolded study of engineering across primary, secondary, and post-secondary education. If a goal of education is to promote the development of more innovative and effective problem-solvers, a coherent study of engineering across $\mathrm{P}-12$ education seems compulsory.

In light of this discussion, the authors believe that scaffolding the instruction and practice of problem framing is necessary. To do so, one recommendation may be to begin first with the problem-driven design approaches of framing a well-defined problem in order to limit potential distractions from solution. In terms of learning outcomes, this can support achieving the Next Generation Science Standard's (NGSS) (2013) engineering design performance expectation that states students, grades K-2, should be able to:

Ask questions, make observations, and gather information about a situation people want to change to define a simple problem that can be solved through the development of a new or improved object or tool.

Then, as students progress toward the end of primary school, they can practice defining problems in a more abstract manner to help meet the NGSS performance expectation for grades 3 though 5 which states that students should be able to:

Generate and compare multiple possible solutions to a problem based on how well each is likely to meet the criteria and constraints of the problem.

Also, as most of the participants' efforts were devoted to just making a prototype without much forethought, it may be beneficial to require approval of a student's design concept prior to making. If all of these practices are maintained, then students may be able to develop the abilities to identify design goals, criteria, and constraints to formulate problem statements that first, help them frame the design scenario as a means to guide their problem solving process and then, remove assumptions limiting solution opportunities by rephrasing a problem statement from multiple perspectives. However, further research into these possibilities is necessary.

Examining the Scaffolding of Engineering Design Experiences The cognitive processing times in this study suggest that between kindergarten and fourth grade, students may develop the cognitive abilities to tackle slightly more complex design problems. However, without the proper scaffolding of design activities from structured to ill-structured problems, students, such as the one fourth grade student discussed earlier, may continue to be distracted by the irrelevant items related to the contextually-rich problem scenarios and thus, be ineffective in identifying and solving the actual problem presented in a design 
activity. These findings suggest the need for further research into students' abilities to transfer ideas, articulate concepts, and progress through design challenges across grade levels. It is possible that the findings from this study may not be replicated with different students, age groups, and participant backgrounds - thus, additional efforts to understand these concepts are encouraged. However, without the presence of a formalized P12 engineering curriculum, and prepared teachers throughout primary schools, students may continue to hold a limited view of engineering (e.g., engineering is simply a craftsoriented subject). As observed in the settings in which this study took place, teachers did not seemed equipped with the knowledge and tools for discussing problem framing and scoping with students and provided little interventions to direct students toward understanding the problem, which may have been evidenced by the limited cognitive effort placed by the participants on Defining Problems. Therefore, it seems that tested and validated guidelines for appropriately scaffolding design experiences may mitigate these concerns with the implementation of engineering design tasks.

Accordingly, the authors created Fig. 6 to provide a sample conceptual model for scaffolding design experiences across the grade levels to potentially help students develop effective design abilities and achieve more "engineering" design capabilities. As the figure depicts, starting in the early grades, students could be provided with structured design problems, that will inherently be inauthentic, to allow them to build upon playful and experimental approaches to design and making. The structured problems can provide experiences for students to achieve some success to begin building their design confidence and making abilities. However, as students develop and their knowledge deepens, the authors posit that they should be provided with more authentic, and less-structured problems, which may provide them with opportunities to learn from failure while also continuing to build design confidence. As students continue to grow and develop more analytic thinking abilities, they could then move from these trial-and-error problem solving approaches to more informed design that includes more calculated engineering design practices - which also necessitates the developmentally appropriate applications of STEM knowledge. In doing such, the authors propose that students can begin to "take the chance out" of designing by narrowing down and refining ideas through informed engineering design practices as opposed to a simple "guess-and-check" or "trial-and-error" approach.

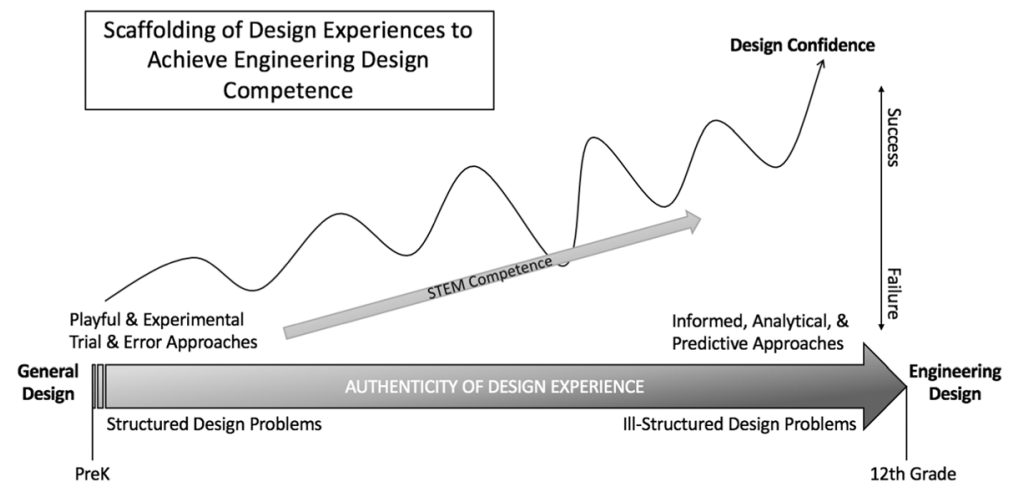

Fig. 6 Conceptual model of scaffolding design experiences across the grade levels to potentially support the development of authentic engineering design capabilities 
Computational Thinking and Scientific Inquiry in Design Another identified area of weakness in the teaching of engineering design at the P-12 level is the appropriate application of computational thinking and scientific inquiry to support the practices of design, particularly at the high school level (National Academy of Engineering and National Research Council 2009). Merrill et al. (2009) posit that the necessary analytical tools for engineering design are not frequently used in classrooms to inform design ideas and as such, they stress the importance of teaching secondary engineering students how to be predictive in designing. While computational and scientific tools and practices may be beyond the capabilities of primary students, it is important to build their foundation for future success in design. In spite of these calls for increased emphasis in this area, studies continue to highlight students' lack of computational, mathematical, and scientific thinking necessary for authentic engineering design (Kelley 2008; Kelley et al. 2010; Kelley and Sung 2017; Wilson et al. 2013). Relatedly, this study found that primary school students rarely engaged in the cognitive strategies for Computing, Experimenting, and Interpreting Data.

Appropriately, Kelley et al. (2010) emphasized the necessity to provide students with more learning opportunities to perform computational/mathematical and scientific inquiry activities within the engineering design process. Although primary students may not be equipped with mathematical or scientific principles for solving authentic problems, it can be helpful for children to experience activities which push them toward the application of fundamental mathematical principles they have learned in class, the use of scientific tools, an emphasis on recording experiment results, and a purposeful effort toward using the resulting data of these actions to inform solutions. A study of fifth grade students conducted by Kelley and Sung (2017) found that when participants were engaged in an engineering design task that was developed to necessitate the application of the mathematical and scientific understandings of the concept of mass, they failed to recall the conservation of mass, compute the mass of their solution, and understand the difference between the concept of mass and volume. However, their study did show that primary students were able to increase the amount of time they dedicated to computational thinking when given additional "transfer" design tasks with embedded mathematics/science activities. Therefore, Kelley and Sung (2017) recommend that primary teachers should provide additional opportunities for students to transfer their knowledge beyond an initial design task. What this effort involves, is developing a "transfer" design task, which is a design problem that is structurally similar to the initial design task but requires students to transfer their learning from the initial task to a novel context. This effort can potentially provide students an opportunity beyond the initial design task, to enhance their use of the computational and scientific mental processes of Computing, Experimenting, and Interpreting Data.

\section{Conclusion}

As the teaching of engineering continues to gain attention in the primary school setting, it becomes fundamental to comprehend the ways in which students cognitively process engineering design tasks to provide effective engineering instruction, establish the adequate scaffolding of engineering design experiences, and enact interventions to 
enhance student design abilities. However, there has been minimal research to provide insight into the range of cognitive processes employed by primary students while designing and making a product, system, or device and limited discussion on effective ways to link design cognition research with practice in engineering education. Moreover, if content standards for engineering are ever to be developed, then use-inspired design cognition research is a necessity (National Academy of Engineering and National Research Council 2010). Consequently, the authors enacted a multiple exploratory case study approach employing the concurrent think-aloud protocol analysis technique to describe the cognitive activity of both kindergarten and fourth grade students as they worked to complete engineering design tasks. This study was driven by the overarching research objectives of (1) identifying ways in which primary students cognitively navigate an engineering design task from design conception through prototype production and (2) determining ways in which to inform P-12 engineering education by examining design cognition in respect of student grade level and design achievement.

To achieve the first research objective, the authors sought to determine what cognitive processes kindergarten and fourth grade students employ to design and make solutions to engineering design problems. To do so, the authors collected and coded video recordings of participants thinking aloud during engineering design tasks. The analysis of the data revealed that the top three most employed mental processes - of the 17 mental processes for technological problem solving (Halfin 1973) - for kindergarten students were Model/Prototype Constructing, Managing, and Observing and the least used mental processes (each consuming less than $2 \%$ of the students design time on average) were Testing, Creating, Measuring, Defining Problems, Questioning, and Predicting. In addition, the mental processes of Computing and Experimenting were not observed in the kindergarten participants. As for the fourth grade participants, the top three most used mental processes were Model/Prototype Constructing, Managing, and Analyzing and least used mental processes (each consuming less than $2 \%$ of the students design time on average) were Creating, Visualizing, Measuring, and Predicting. Also, similar to the kindergarten students, the mental processes of Computing and Experimenting were not observed being used by the fourth grade participants. Lastly, the analysis revealed a tendency for both groups of students to allocate the largest portion of their cognitive activity toward manipulating materials to produce a physical prototype rather than problem framing, information gathering, and generating design concepts. While fourth grade students in this study dedicated significantly more cognitive effort to Defining Problems than kindergarten students, the two groups, overall, devoted limited time to analyzing and defining the given problems to generate refined solution ideas.

To achieve the second research objective, the authors sought to examine potential relationships between the design cognition of kindergarten and fourth grade students as well as identify any potential cognitive indicators for design achievement. To do so, the design cognition data of the two participant groups were compared along with the evaluations of their design work. The analysis revealed significant differences between kindergarten and fourth grade participants with regard to the amount of time various cognitive processes were employed to complete a design task. Fourth grade students dedicated significantly more time to the mental processes of Creating, Defining Problems, Measuring, and Testing than kindergarten students. The greatest difference 
in cognitive processing time between the two groups was found in the mental process of Testing. Accordingly, the observations of these students showed that most of the kindergarten participants ended their design session once a prototype was made and essentially no prototype revisions were enacted. However, the fourth grade participants did showcase the iterative design practices related to Testing, which was demonstrated by them examining the workability and/or durability of their prototypes and then attempting to improve their results. In addition, when examining the design protocols along with the evaluations of the student design portfolios, it was found that more time devoted to the cognitive process of Managing could be a significant predictor of a poorer design ranking. This result was seen as a potential effect of limited student abilities in regard to problem framing and scoping. However, the cognitive processes of Creating, Defining Problems, Interpreting Data, Measuring, Observing, Predicting, Testing, and Visualizing, while not statistically significant, were related to a better design ranking.

Based on this analysis, several recommendations for educational practice and future research were discussed. First, to help aid in improving student design abilities, a recommendation could be to provide students with developmentally appropriate instruction related to problem framing and scoping. Initially, engineering design tasks may be too perceptually rich for kindergarten students and distract them from the learning objective of the assignment. Also, their level of experience may limit them from understanding the criteria for the actual design task and result in them physically making objects that are irrelevant to solving the problem. Perhaps the results may even indicate that there is an age where open-ended design tasks are not cognitively appropriate for learners; specifically, if these tasks are used for learning specific STEM concepts rather than just a fun reprieve from the "normal" classroom environment. However, if appropriate attention is given to teaching young children problem-driven design approaches and the practices of defining the problem, determining the criteria and constraints, and identifying clear goals for developing a solution, rather then immediately making prototypes, then students may be able to better manage their design process and ultimately, produce enhanced solutions. Also, the scaffolding of instruction from well-defined problems to more authentic, ill-defined problems could be recommended as a means to help transition students to more informed engineering design practices. Lastly, the participants in this study did not engage in the cognitive strategies for Computing, Experimenting, and Interpreting Data, which can be critical for advancing a student design capabilities as well as enabling their learning of science and mathematics. Therefore, a recommendation from Kelley and Sung (2017) of providing students with math/science embedded "transfer" design tasks that require them to translate their learning from an initial design task to novel contexts seems beneficial for increasing a their use of these cognitive processes.

It is important to note that this study was exploratory in nature and as such, has limitations. Therefore, the authors recommend that the research methods presented in this study be enacted with larger sample sizes of groups of various ages and experience levels. These further investigations can help identify potential cognitive pain points in the design practices of students in an effort to provide support for informing the implementation of engineering at the P-12 level and developing strategies to help improve student design achievement. As engineering design-based activities continue to be touted as a preferred approach to teaching STEM-related content and practices, it 
is important that further attention is given to how students develop and learn design as well as function within design environments.

\section{Compliance with Ethical Standards}

Conflict of Interest On behalf of all authors, the corresponding author states that there is no conflict of interest.

\section{References}

Atman, C. J., \& Bursic, K. M. (1998). Verbal protocol analysis as a method to document engineering student design processes. Journal of Engineering Education, 87(2), 121-132.

Bartholomew, S. R. (2017). Assessing open-ended design problems. Technology and Engineering Education Teacher, 76(6), 13-17.

Bartholomew, S. R., Strimel, G. J., \& Jackson, A. (2017). A comparison of traditional and adaptive comparative judgment assessment techniques for freshman engineering design projects. International Journal of Engineering Education, 34(1), 20-33.

Bartholomew, S. R., Strimel, G. J., \& Yoshikowa, E. (2018). Using adaptive comparative judgment for student formative feedback and learning during a middle school open-ended design challenge. International Journal of Design \& Technology Education.

Berland, L. K. (2013). Designing for STEM integration. Journal of Pre-College Engineering Education Research, 3(1), 22-31.

Berland, L. K, \& Busch, K. (2012). Negotiating STEM epistemic commitments for engineering design challenges. In American Society for Engineering Education 2012 Annual Conference and Exposition, Conference Proceedings.

Blanchard, P., \& Suhr, J. (1999). My bug box. Katonah: Richard C. Owen Publishers, Inc.

Capobianco, B. M., French, B. F., \& Diefes-Dux, H. A. (2012). Engineering identity development among preadolescent learners. Journal of Engineering Education, 101(4), 698-716.

Carr, R. L., Bennett IV, L. D., \& Strobel, J. (2012). Engineering in the K- 12 STEM standards of the 50 U.S. states: An analysis of presence and extent. Journal of Engineering Education, 101(3), 539-564.

Cohen, D. (2002). How the child's mind develops? London: Routledge.

Cohen, B. H. (2007). Explaining psychological statistics (3rd ed.). New York: John Wiley and Sons.

Cross, N. (2001). Designerly ways of knowing: Design discipline versus design science. Design Issues, 17(3), 49-56.

Cross, N. (2004). Expertise in design: An overview. Design Studies, 25(5), 427-441.

Crossland, J. (2015). Is Piaget wrong? Primary Science, 137, 30-32.

Cunningham, C. M. (2009). Engineering is elementary. The Bridge, 30(3), 11-17.

Cunningham, C. M., \& Hester, K. (2007). Engineering is Elementary: An Engineering and Technology Curriculum for Children. Paper presented at ASSE Annual Conference and Exposition, Honolulu, HI. Retrived from http://www.eie.org/sites/default/files/research_article/research_file/ac2007full8.pdf

Dutson, A., Todd, R., Magleby, S., \& Sorensen, C. (1997). A review of literature on teaching engineering design through project-oriented capstone courses. Journal of Engineering Education, 86(1), 17-28.

Dym, C. L., Agogino, A. M., Eris, O., Frey, D. D., \& Leifer, L. J. (2005). Engineering design thinking, teaching, and learning. Journal of Engineering Education, 94(1), 103-120.

Engineering Accreditation Commission. (2016). Criteria for accrediting engineering programs. Baltimore: Accreditation Board for Engineering and Technology Retrieved from http://www.abet.org/wpcontent/uploads/2016/12/T001-17-18-ETAC-Criteria-10-29-16.

Goldstone, R. L., \& Sakamoto, Y. (2003). The transfer of abstract principles governing complex adaptive systems. Cognitive Psychology, 46(4), 414-466.

Grubbs, M. E. (2013). Bridging design cognition research and theory with teaching and learning. New Zealand: Published Proceedings of the Pupil's Attitudes Toward Technology International Conference, Christ Church.

Grubbs, M. E. (2016). Further characterization of high school pre-and non-engineering students' cognitive activity during engineering design (Doctoral dissertation). Retrieved from ProQuest Dissertations and Theses database. (UMI No. 3662376). 
Grubbs, M. E., \& Strimel, G. (2015). Engineering design: The great integrator. Journal of STEM Teacher Education, 50(1), 77-90.

Grubbs, M. E., \& Strimel, G. J. (2016). Cognitive research: Transferring theories and findings to k-12 engineering educational practice, American Society for Engineering Education 103rd Annual Conference and Exposition. New Orleans, LA, June 26, 2016.

Halfin, H. H. (1973) Technology: A process approach. (Doctoral dissertation, West Virginia University, 1973) Dissertation Abstracts International, (1) 1111A.

Hall, M. (2006). Dragonflies. Mankato: Capstone Press.

Hammack, R., Ivey, T. A., Utley, J., \& High, K. A. (2015). Effect of an engineering camp on students' perceptions of engineering and technology. Journal of Pre-College Engineering Education Research, 5(2), 10-21. https://doi.org/10.7771/2157-9288.1102.

Hegedus, T. (2014). Engineering education for youth: Diverse elementary school students' experiences with engineering design (Doctoral dissertation). Retrieved from http://eie.org/sites/default/files/research article/research_file/hegedus-dissertation-final-dec2014.pdf.

Hill, R. B., \& Wicklein, R. C. (1999). A factor analysis of primary mental processes for technological problem-solving. Journal of Industrial Teacher Education, 36(2), 83-100.

International Technology and Engineering Educators Association (ITEA/ITEEA). (2000/2002/2007). Standards for technological literacy: Content for the study of technology. Reston, VA: Author.

Kaminski, J. A., Sloutsky, V. M., \& Heckler, A. (2009). Transfer of mathematical knowledge: The portability of generic instantiations. Child Development Perspectives, 3(3), 151-155.

Kelley, T. R. (2008). Cognitive processes of students participating in engineering-focused design instruction. Journal of Technology Education, 19(2), 50-64.

Kelley, T., \& Sung, E. (2017). Examining elementary school students' transfer of learning through engineering design using think-aloud protocol analysis. Journal of Technology Education, 28(2), 83-108.

Kelley, T. R., Brenner, D. C., \& Pieper, J. T. (2010). Two approaches to engineering design: Observations in stem education. Journal of stem Teacher Education, 47(2), 5-40.

Kelley, T. R., Capobianco, B. M., \& Kaluf, K. J. (2015). Concurrent think-aloud protocols to assess elementary design students. International Journal of Technology and Design Education, 25(4), 521-540.

Kimbell, R. (2012). Evolving project e-scape for national assessment. International Journal of Technology \& Design Education, 22, 135-155.

Kolpin, M. (2014). Great women of the civil war. Mankato: Capstone Press.

Kruger, C., \& Cross, N. (2006). Solution driven versus problem driven design: Strategies and outcomes. Design Studies, 27(5), 527-548.

Lammi, M. D., \& Becker, K. (2013). Engineering design thinking. Journal of Technology Education, 24(2), 55-77.

Lammi, M. D. \& Gero, J. S. (2011). Comparing design cognition of undergraduate engineering students and high school pre-engineering students. Paper presented at the 2011 Frontiers in Education Conference, Rapid City, SD.

Lead States, N. G. S. S. (2013). Next generation science standards: For states, by states. Washington, DC: National Academies Press.

McCullar, H. (2015). Am I really teaching engineering to elementary students? Science \& Children, $52(7), 80-84$.

McHugh, M. (2012). Interrater reliability: The kappa statistic. Biochemia Medica, 22(3), 276-282.

Mechisedech, K. (2011). The terrible, awful civil war. Mankato: Capstone Press.

Mentzer, N. (2014). Team based engineering design thinking. Journal of Technology Education, 25(2), 52-72.

Mentzer, N., Becker, K., \& Sutton, M. (2015). Engineering design thinking: High school students' performance and knowledge. Journal of Engineering Education, 104(4), 417-432.

Merrill, C., Custer, R. L., Daugherty, J., Westrick, M., \& Zeng, Y. (2009). Delivering core engineering concepts to secondary level students. Journal of Technology Education, 20(1), 48-64.

National Academy of Engineering \& National Research Council. (2009). Engineering in K-12 education: Understanding the status and improving the prospects. Washington, DC: The National Academies Press.

National Academy of Engineering \& National Research Council. (2010). Standards for K-12 engineering education? Washington, DC: The National Academies Press.

National Academy of Engineering \& National Research Council. (2014). STEM integration in K-12 education: Status, prospects, and an agenda for research. Washington, DC: National Academies Press.

NGSS Lead States. (2013). Next generation science standards: For states, by states. Washington, DC: National Academies Press.

Polacco, P. (1994). Pink and say. New York: Babushka Press. 
Purcell, A. T., Gero, J. S., Edwards, H., \& McNeill, T. (1996). The data in design protocols: The issue of data coding, data analysis in the development of models of the design process. In N. Cross, H. Christiaans, \& K. Dorst (Eds.), Analysing design activity (pp. 225-252). Chichester: John Wiley.

Seery, N., Buckley, J., Doyle, A., \& Canty, D. (2016). The validity and reliability of Adaptive Comparative Judgements in the assessment of graphical capability. In Proceedings of the 71st Mid-Year Conference of the Engineering Design Graphics Division (pp. 104-109).

Sloutsky, V. M., Kaminski, J. A., \& Heckler, A. F. (2005). The advantage of simple symbols for learning and transfer. Psychonomic Bulletin \& Review, 12(3), 508-513.

Strimel, G. J. (2014). Engineering design: A cognitive process approach (Doctoral dissertation). Retrieved from ProQuest Dissertations and Theses database. (UMI No. 3662376).

Strimel, G. J., Grubbs, M. E, \& Wells, J. G. (2016). Engineering education: A clear decision. Technology and Engineering Teacher, 76(4), 18-24.

Strimel, G. J., Bartholomew, S. R., Kim, E., \& Cantu, D. V. (2018). Examining engineering design cognition with respect to student performance. International Journal of Engineering Education. 34(6), 1-20.

Sutherland, P. (1992). Cognitive development today : Piaget and his critics. London: P. Chapman Pub.

Sweeney, A. (2010). Toads. Mankato: Capstone Press.

Weiten, W. (1992). Psychology: Themes and variations (2nd ed). California: Brooks Cole Publishing Company.

Welch, M., \& Lim, H. S. (2000). The strategic thinking of novice designers: Discontinuity between theory and practice. Journal of Technology Studies, 26(2), $34-44$.

Wicklein, R. C., \& Rojewski, J. W. (1999). Toward a "unified curriculum framework" for technology education. Journal of Industrial Teacher Education, 36(4), 38-56.

Wilson, A. A., Smith, E. R., \& Householder, D. L. (2013). High school students' cognitive activity while solving authentic problems through engineering design processes. Atlanta: Published Proceedings of the American Society of Engineering Education. 